\title{
Photodynamic Disinfection of Dentures
}

\author{
Angelina Pecheva Vlahova ${ }^{1, *}$, Christo Kalchev Kissov ${ }^{1}$, Elka Vakrilova Popova ${ }^{2}$, Georgi Rangelov Todorov ${ }^{1}$ \\ ${ }^{1}$ Department of Prosthetic Dental Medicine, Faculty of Dental Medicine, Plovdiv, Bulgaria \\ ${ }^{2}$ Department of Periodontology and Oral Mucosa Diseases, Faculty of Dental Medicine, Plovdiv, Bulgaria \\ *Corresponding author: a_vlahova@yahoo.com \\ Received December 28, 2012; Revised May 05, 2013; Accepted May 10, 2013
}

\begin{abstract}
Photodynamic disinfection is a new method for cleaning dentures of edentulous patients. It is based on the photochemical action of a photoactive dye (photosensitizer), activated by proper light in the presence of oxygen. The mechanism of photosensitization directs the generation of reactive oxygen species that destroy the pathogens. A pilot study of photodynamic disinfection of dentures was carried out. Four different photosensitizers susceptible to red light were used for the investigation. Results showed that photodynamic disinfection is an effective method for inactivation of pathogens on dentures as a part of prevention of cross-contamination in dental office. It also can be used as a part of the therapy of denture stomatitis.
\end{abstract}

Keywords: photodynamic disinfection, dentures, prevention, cross-contamination

\section{Introduction}

Photodynamic therapy (PDT) is a method for inactivation of pathogenic and tumor cells, using photoactive dye (photosensitizer, PS), which is sensitive to light with specific wavelength $[1,2,3,4,5]$. The energy transfer from PS to the available oxygen leads formation of extremely toxic oxygen species, as free radicals and singlet oxygen ${ }^{1} \mathrm{O}_{2}$ that deactivate pathogens $[6,7,8,9]$. PDT is high selective to pathogenic cells, because PS has the ability to identify them and to conglomerate in them [3].

Three groups of photosensitizers were developed until now: group of the dyes (metal phthalocyanines are included here), photofrin and its derivatives and furocoumarines [10]. Most of the PS are sensitive to red light between 630 and $700 \mathrm{~nm}[11,12]$, but some of them can be activated by blue light $380-500 \mathrm{~nm}$ and ultraviolet (UV) light < 400nm [13].

Initially photodynamic therapy was used in the oral cavity in the middle 80's of the last century [4]. In the Prosthetic Dentistry PDT can be used as photodynamic disinfection (PDD) of dental impressions and dentures for prevention of cross-infection between dentist, dental technician and patient, and also as a part of the therapy of denture stomatitis $[14,15,16]$.

The aim of the present study was to evaluate efficacy of the photodynamic disinfection as a method for cleansing of contaminated with pathogenic microorganisms dentures of edentulous patients.

\section{Materials and Methods}

Four different photosensitizers susceptible to red light $550-750 \mathrm{~nm}$ (Figure 1) were used for the investigation: three metal phthalocyanines - gallium (GaPc1), zinc
(ZnPc1) and silicon (SiPc1), and FotoSan (FS) (CMS Dental), based on Tylenol. The chemical structure of the metal phthalocyanines is shown on Figure 2. The metal phthalocyanines were used as a solutions in concentrations $5 \mu \mathrm{l} / \mathrm{ml}$ in $0,9 \% \mathrm{NaCl}$. PS FotoSan (CMS Dental) has three manufacture consistencies - liquid (low), semiliquid (medium) and gel (high). We used liquid (low) consistence for the investigation.

147 acrylic dentures of edentulous patients from the Department of Prosthodontics in the Faculty of Dental Medicine - Plovdiv, Bulgaria were cleaned by the new method of photodynamic disinfection (PDD). Six different kinds of microorganisms were isolated from the dentures before disinfection: $\alpha$-haemolythical Streptococcus viridans, coagulasa-negative Staphylococcus, nonpathogenic Neisseria, Pneumococcus, Candida species and Corynebacteria. First each denture was washed with water from the sink and was brushed mechanically with toothbrush that eliminated the biofilm from the surfaces. After that it was immersed in PS for 10 minutes and then it was irradiated for 10 minutes in apparatus for photodynamic disinfection of dental impressions and prosthetic constructions - illumination device (LED lamp), which presents light box with $625 \mathrm{~nm}$ red light - diodes [14].

\section{Results}

91 of 147 dentures were totally sterile after disinfection. From all 147 dentures 41 were treated with PS GaPc1, 36 were treated with PS ZnPc1, 37 were treated with PS SiPc1 and 33 were treated with PS FotoSan (FS).

Candida species were isolated from 45 of 147 dentures before disinfection. The effectiveness of PDD with PS GaPc1 and ZnPc1 is $100 \%$, with PS SiPc1 is $92 \%$ and with PS FS is $97 \%$.

$\alpha$-haemolythical Streptococcus viridans was isolated from all of 147 dentures. The efficacy of PDD with PS 
GaPc1 is $73 \%$, with PS ZnPc1 - 69\%, with PS SiPc1 $81 \%$ and with PS FS $-73 \%$.

Coagulasa negative Staphylococcus were presented in 123 of 147 dentures before disinfection. The effectiveness of PDD with different PS was: $85 \%$ with PS GaPc1, 81\% with PS ZnPc1, 70\% with PS SiPc1 and $76 \%$ with PS FS.

Non-pathogenic Neisseria were cultivated from 93 of 147 dentures. After PDD the effectiveness was: $90 \%$ with PS GaPc1, 100\% with PS ZnPc1, 92\% with PS SiPc1 and 91\% with PS FS.

Pneumococci were isolated from 35 of 147 dentures before disinfection. After PDD with all the tested PS the effectiveness is $100 \%$.

We determinated Corynebacteria on 18 of 147 dentures before disinfection. PDD showed $93 \%$ effectiveness with PS GaPc1 and $100 \%$ with PS ZnPc1, SiPc1 and FS.

The percent ratios of the different microorganisms tested with different PS by the method of PDD are shown at Figure 3.

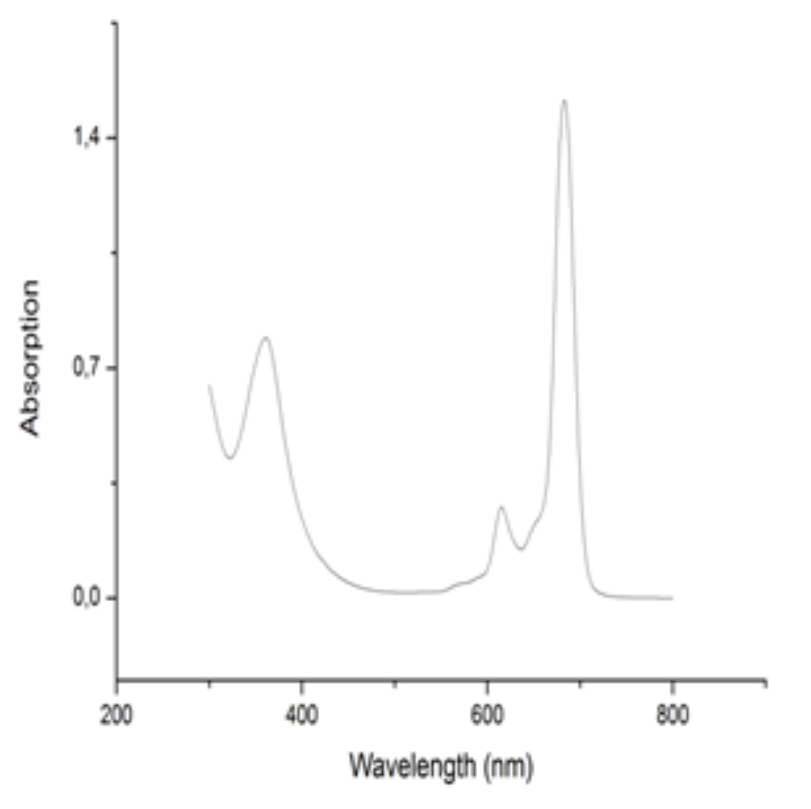

Figure 1. Absorption spectrum of red light - activated photosensitizers

\section{Discussion}

Bacterial adhesion on denture surfaces leads to biofilm formation. Biofilm is an organized cooperation of microorganisms on a surface, usually moist $[17,18]$. Its formation makes Candida, an infection on acrylic dentures, exceptionally resistant [19,20]. Gram-positive Candida species, which are basic agents of denture stomatitis, especially $C$. albicans, are difficult for inactivation by PDD, because they have large cell with nucleus membrane $[21,22]$.

Candida colonization on dentures of edentulous patients depends of different factors. $C$. tropicalis has the best adhesion, followed by $C$. glabrata, $C$. dubliniensis and $C$. albicans [23]. Acrylic resin adhesion of Candida albicans varies in vitro as a result of the action of the residual monomer left after the polymerization [24]. Saliva decreases the bacterial adhesion, but the increased number of microorganisms advantages the process [25].
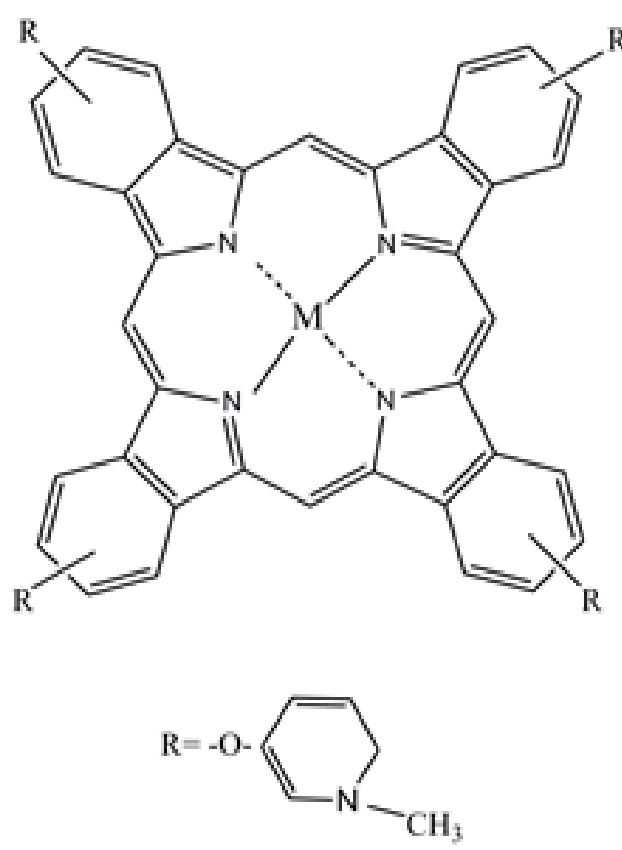

\section{$\mathrm{M}=\mathrm{Zn}(\mathrm{II}), \mathrm{Ga}(\mathrm{III})$ and $\mathrm{Si}(\mathrm{IV})$}

Figure 2. Chemical structure of the used metal phthalocyanines

Candida species colonized dentures and oral cavity may cause infection of the fingertips of the patient himself and of the dentist or the dental technician [26]. This is the reason for American Dental Association (ADA) to recommend that every patient should be treated as a source of infection [27].

Regarding to Candida species photodynamic disinfection (PDD) of dentures showed $100 \%$ effectiveness with PS GaPc1 and ZnPc1. The lower degree of disinfection with the other two PS can be explained with differences in the chemical structure. In our previous in vitro study [28] we ascertained the fact that PS GaPc1 and ZnPc1 have higher antimicrobial activity than SiPc1 in reference to three test microorganisms: methicillin-resistant Staphylococcus aureus (MRSA), Pseudomonas aeruginosa and $C$. albicans.

According to Konopka and Goslinski, 2007 [3] porous external layer of the cytoplasm membrane of the Gram positive bacteria facilitates the PS diffusion in the cell. On the other hand the cell wall of Gram negative bacteria is an effective barrier between the cell and the environment. That is the reason for the higher efficacy of PDD against Gram positive than Gram negative microorganisms [5].

$\alpha$-haemolythical Streptococcus viridans is one of the main agents that cause infectious endocarditis $(60 \%$ of the cases), caries and gingivitis [29]. We isolated it in all 147 examined dentures before PDD. The percent's of the effectiveness of the disinfection with different PS were close (between 69 and 81\%). Biofilm formation made difficult PS and red light penetration in depth and decreased the effect of PDD.

We determinated the presence of coagulasa negative Staphylococcus in 123 of 147 dentures before disinfection. These bacteria are classified at coagulasa positive and negative, because of their ability to produce the enzyme coagulasa that forms thrombi in blood. Coagulasa negative are S. epidermidis S. saprophyticus S. lugdunensis, $S$. 
schleiferi, S. caprae [30]. The percent of efficacy of PDD with different PS against these microorganisms is high (70 $-85 \%)$.

Neisseria are a big genus Gram negative bacteria, colonized animals and human mucosa. Only 2 of 11 human species are pathogenic - N. meningitidis and $N$. gonorrhoeae [31]. Effectiveness of inactivation after PDD is really high $(90-100 \%)$.

Pneumococcus is Gram positive $\alpha$-haemolythical anaerobe. Except pneumonia it may cause many other infections such as: acute sinusitis, otitis, meningitis, bacteremia, sepsis, pericarditis, peritonitis and brain abscess [32]. Against these bacteria PDD is $100 \%$ effective method.
Some Corynebacteria are a part of the normal oral flora and others are extremely pathogenic like $C$. diphtheriae, which causes diphtheria [33]. Our method of PDD is $100 \%$ effective against isolated Corynebacteria with PS ZnPc1, SiPc1 and FS. Only PS GaPc1 showed lower degree of disinfection (93\%).

The effectiveness of photodynamic disinfection depends of some other factors: the age of dentures, oral hygiene habits of the patient and the diet. Biofilm formations on dentures make the disinfection extremely difficult.

Mechanical brushing with toothbrush and water from the sink helps cleaning the big amounts of biofilm on denture surfaces and supports the next PDD, because of easier penetration of PS and the light in depth.

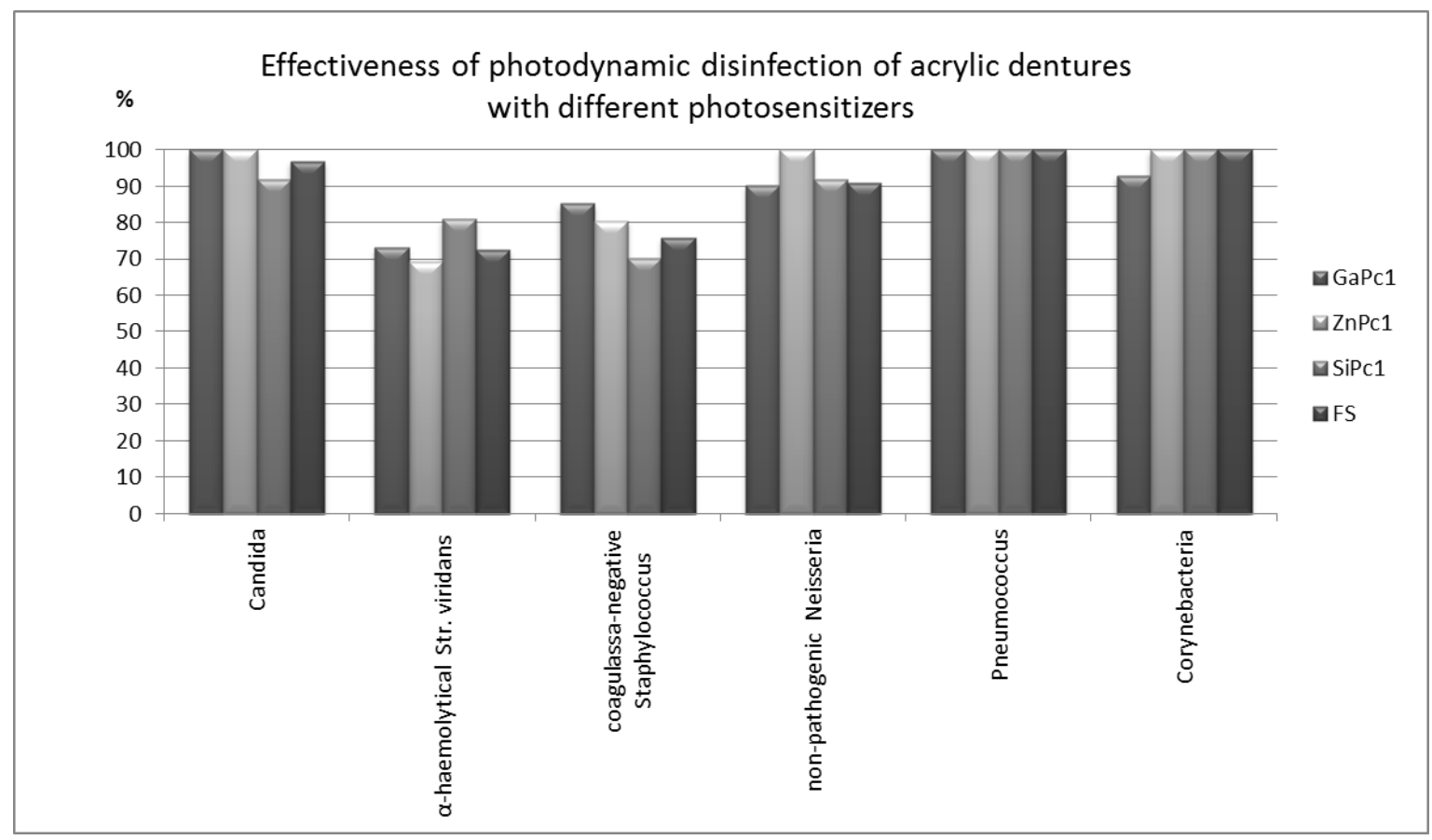

Figure 3. Effectiveness of photodynamic disinfection of acrylic dentures with different photosensitizers

\section{Conclusion}

Every denture is soiled with saliva, gingival fluid, and dental plaque. All body fluids are contaminated with opportunist and pathogenic bacteria. These facts give an opportunity the denture to become a source of crossinfection.

This study suggests a new alternative method for disinfection of dentures by using photodynamic therapy as prevention of cross-contamination between patient, dentist and dental technician. Disinfection of dentures is also a main part of the therapy of denture stomatitis.

\section{Acknowledgements}

The work was supported of the National Science Fund, Bulgaria (Grant DO-02-177/08).

\section{Statement of Competing Interests}

The authors have no competing interests (financial or others) that may have influenced the study or the conclusions drawn from the study.

\section{List of Abbreviations}

$\begin{array}{ll}\text { PDT } & \text { Photodynamic Therapy; } \\ \text { PDD } & \text { Photodynamic Disinfection; } \\ \text { PS } & \text { photosensitizer; } \\ \text { GaPc1 } & \text { gallium phthalocyanines 1; } \\ \text { ZnPc1 } & \text { zinc phthalocyanines 1; } \\ \text { SiPc1 } & \text { silicon phthalocyanines 1; } \\ \text { FS } & \text { FotoSan (CMS Dental). }\end{array}$

\section{References}

[1] Donnely R, P McCarron, M Tunney. Antifungal photodynamic therapy. Microbiological Research, 163(1), 1-12, 15 Jan 2008

[2] Kömerik N. Photodynamic therapy as an alternative antimicrobial modality for oral infections. Journal of Environmental Pathology, Toxicology and Oncology. Iss 1-2, pp 546, Vol. 25, 2006.

[3] Konopka K, Goslinski T. Photodynamic therapy in dentistry. J Dent Res; 86(8): 694-07, 2007.

[4] Konopka K, Goslinski T. Prospects for photodynamic therapy in dentistry.

http://www.photonics.com/Content/ReadArticle.aspx?ArticleID=3 4401 . 
[5] Mantareva, V., V. Kussovski, I. Angelov, D. Wöhrle, R. Dimitrov, E. Popova, S. Dimitrov. Non - aggregated Ga(III)-phthalocyanines in the photodynamic inactivation of planktonic and biofilm cultures of pathogenic microorganisms. Photochem. Photobiol. Sci., 10, 91-102, 2011.

[6] Abdel-Shafi A, D Worrall. Mechanism of the excited singlet and triplet states quenching by molecular oxygen in acetonitrile. Journal of Photochemistry and Photobiology A: Chemistry. 172, 170-179, 2005.

[7] Fernandez JM, MD Bilgin, LI Grossweiner. Singlet oxygen generation by photodynamic agents. Journal of Photochemistry and Photobiology B: Biology, 37, 131-140, 1997.

[8] Mitra S, T Foster. Photochemical oxygen consumption sensitized by a Porphyrin phosphorescent probe in two model systems. Biophysical Journal, 78, 2597-2605, May 2000.

[9] Wainwright M. Photodynamic antimicrobial chemotherapy (PACT). Journal of Antimicrobial Chemotherapy, 42, 13-28, 1998.

[10] Josefsen L, Boyle R. Photodynamic therapy and the development of metal-based photosensitisers. Review article. Metal-Based Drugs, Vol. 2008; Article ID 276109, 24 pages.

[11] Canavesi C, F Fournier, W Cassarly, T Foster, J Rolland. Illumination devices for photodynamic therapy of the oral cavity. Biomed Opt Express. 1(5): 1480-1490, 2010.

[12] König K, M Teschke, B Sigusch, E Glockmann, S Eick, W Pfister. Red light kills bacteria via photodynamic action. Cell Mol Biol (Noisy-le-grand). 46 (7): 1297-303, Nov 2000.

[13] Bouillaguet S, Owen B, Wataha J, Campo M, Lange N, Schrenzel $\mathrm{J}$. Intracellular reactive oxygen species in monocytes generated by photosensitive chromophores activated with blue light. Dent Mater, 24, 1070-1076, 2008.

[14] Vlahova A. Possibilities of application of photodynamic therapy in prosthetic dental medicine. PhD dissertation, Plovdiv, Bulgaria 2012.

[15] Vlahova. A., Chr. Kissov, E. Popova, I. Haydushka, V. Mantareva. A new method for photodynamic disinfection of prosthetic constructions and impressions in prosthetic dentistry. Folia Medica, Plovdiv, Bulgaria, 54(1), 51-57, 2012.

[16] Vlahova A., Chr. Kissov, E. Popova. Photodynamic disinfection of dental impressions as a new competitive method to the conventional cleansing procedures. Journal of Analytical Oncology, 1(2), 187-191, Dec 2012.

[17] Overman P. Biofilm: a new view of plaque. The Journal of Contemporary Dental Practice, 1(3), Summer Issue, 1-8, 2008.

[18] Avon SL, JP Goulet, N Deslauriers. Removable acrylic resin disk as a sampling system for the study of denture biofilms in vivo. J Prosthet Dent; 97: 32-8, 2007.
[19] Emami, E., J. Seguin, P. Rompre, L. Koninck, P. de Grandmont, J. Barbeau. The relationship of myceliated colonies of Candida albicans with denture stomatitis: An in vivo/in vitro study. Int $\mathbf{J}$ Prosthodont; 20: 514-520, 2007.

[20] Zanin ICJ, MM Lobo, LKA Rodrigues, LAF Pimenta, JF Hofling, RB Goncalves. Photosensitization of in vitro biofilms by toluidine blue O combined with a light-emitting diode. Eur J Oral Sci; 114: 64-69, 2006

[21] Bliss JM, CE Bigelow, TH Foster, CG. Haidaris. Susceptibility of Candida species to photodynamic effects of Photofrin. Antimicrobial Agents and Chemotherapy, 48(6): 2000-2006, June 2004.

[22] Mima, E., A. Pavarina, L. Dovigo, C. Vergani, C. Costa, C. Kurachi, V. Bagnato. Susceptibility of Candida albicans to photodynamic therapy in a murine model of oral candidosis. Oral Surg Oral Med Oral Pathol Oral Radiol Endod; 109: 392-401, 2010.

[23] Moura J., W. da Silva, T. Pereira, A. Cury, R. Garcia. Influence of acrylic resin polymerization methods and saliva on the adherence of four Candida species. J Prosthet Dent; 96: 205-11, 2006.

[24] Serrano-Granger C., R. Cerero-Lapiedra, J. Campo-Trapero, J. Del Rio-Highsmith. In vitro study of the adherence of Candida albicans to acrylic resins: Relationship to surface energy. Int J Prosthodont; 18: 392-398, 2005.

[25] Pereira T., A. Cury, M. Cenci, R. Rodrigues-Garcia. In vitro Candida colonization on acrylic resins and denture liners: influence of surface free energy, roughness, saliva, and adhering bacteria. Int J Prosthodont; 20: 308-310, 2007.

[26] Darwazeh A, S Al-Refai, S Al-Mojaiwel. Isolation of Candida species from the oral cavity and fingertips of complete denture wearers. J Prosthet Dent; 86: 420-3, 2001

[27] ADA Council on Scientific Affairs and ADA Council on Dental Practice. Infection control recommendations for the dental office and the dental laboratory. JADA, 127: 672-680, May 1996.

[28] Popova E., Sl. Dimitrov, Chr. Kissov, A. Vlahova, I. Angelov, V. Mantareva, V. Kussovski. Photodynamic destruction of Candida albicans on dentures with porphyrin and phthalocyanines. 14-th Congress of BaSS. 9-th Scientific Congress of BgDA. Varna, Bulgaria, 6-9 May 2009.

[29] http://en.wikipedia.org/wiki/Streptococcus_viridans.

[30] http://en.wikipedia.org/wiki/Staphylococcus.

[31] http://en.wikipedia.org/wiki/Neisseria.

[32] http://en.wikipedia.org/wiki/Pneumococcus.

[33] http://en.wikipedia.org/wiki/Corynebacterium. 